\title{
Biphasic turbulence
}

Nat. Commun. 10, 3333 (2019)

Turbulent flow is an effective way to enhance heat transfer rates from a hot surface to a fluid. However, in classical thermal turbulence heat exchange is limited by the mixing between the turbulent bulk flow and the boundary layer. Although different strategies have been proposed to overcome this limit, heat transfer has been enhanced up to $50 \%$ at best while requiring system modifications that are not always compatible with existing heating and cooling devices, such as closed-system heat exchangers. Now, Chao Sun and colleagues in China and the USA achieve a 500\% enhancement in heat transfer compared to a standard turbulent system by exploiting biphasic heat-carrier elements.

The researchers introduce a small quantity, $1 \%$ by volume, of a hydrofluoroether liquid to a water-based thermal convection system. The working fluid is confined by two copper plates that are held at temperatures above and below the boiling point of the hydrofluoroether liquid. This results in a dynamic evaporationcondensation cycle with vapour bubbles detaching from the bottom plate and gradually condensing into liquid droplets - a biphasic heat-carrier element that releases heat in the bulk - when reaching the top cold plate. When the temperature of the bottom plate is raised $10 \mathrm{~K}$ above the boiling point, the researchers observe a marked increase in the collective large-scale circulation velocity compared to classical turbulence, which is attributed to fast and chaotic mixing induced by the biphasic particles. Additionally, after releasing heat in the bulk the biphasic particles condense at the top plate and move downward, bringing the system to its initial state.

Giulia Tregnago

Published online: 13 August 2019

https://doi.org/10.1038/s41560-019-0454-7 